\title{
Three-Dimensional Simulation of One-Dimensional Transport in Silicon Nanowire Transistors
}

\author{
Gianluca Fiori and Giuseppe Iannaccone
}

\begin{abstract}
We present a simulation study of silicon nanowire transistors, based on an in-house code providing the self-consistent solution of Poisson, Schrödinger, and continuity equations on a generic three-dimensional domain. The main assumption, based on the very small nanowire cross section considered, is that an adiabatic approximation can be applied to the Schrödinger equation, so that transport occurs along one-dimensional subbands. Different subband transport models are considered, such as ballistic transport, either including quantum tunneling or not, and drift-diffusion. We show that nanowire transistors exhibit good control of short channel effects, and that barrier tunneling is significant in the strong inversion regime even for longer devices, while it is significant in subthreshold only for the shortest channel lengths. Finally, we show that a subband-based transport model allows to reach a very good trade off between physical accuracy of the simulation and computing time.
\end{abstract}

Index Terms-Ballistic transport, drift-diffusion, Poisson/ Schrödinger, silicon nanowire transistor, three-dimensional, 1-D subbands.

\section{INTRODUCTION}

$\mathbf{S}$ ILICON nanowire transistors (SNWTs) are considered an interesting alternative architecture to the conventional planar technology for devices at the end of the ITRS roadmap [1]-[3], because of their improved electrostatic control of the channel via the gate voltage and the consequent suppression of short channel effects [4]-[7].

Actual SNWTs are intrinsically three-dimensional, and spatial symmetries in nonidealized devices cannot be invoked to reduce the spatial degrees of freedom. In addition, it is well known that the behavior of field-effect transistors is dominated by electrostatics, which has therefore to be accurately simulated in order to reproduce the device electrical behavior.

For this reason, simulations based on the self-consistent solution of Poisson, Schrödinger and transport equations in three dimensions represent a required tool to understand device behavior and extract design guidelines. The solution of the

Manuscript received August 25, 2006; revised March 1, 2007. This work was supported in part by the European Commission's Information Society Technologies Programme, under PULLNANO Project Contract IST-026828, and in part by the European Science Foundation EUROCORES Programme Fundamentals of Nanoelectronics, through funding from the Consiglio Nazionale delle Ricerche (awarded to IEEIIT-PISA) and the European Commission Sixth Framework Programme, under Project Dewint (Contract ERAS-CT-2003-980409). The review of this paper was arranged by Associate Editor R. Lake.

The authors are with the Dipartimento di Ingegneria dell'Informazione, Università di Pisa, 56122 Pisa, Italy (e-mail: g.fiori@ iet.unipi.it; g.iannaccone@ iet. unipi.it).

Color versions of one or more of the figures in this paper are available online at http://ieeexplore.ieee.org.

Digital Object Identifier 10.1109/TNANO.2007.896844
Schrödinger equation, in particular, provides the correct density of states that is significantly different from the bulk density of states, due to transversal quantum confinement, and has an obvious direct impact on the charge density.

Semiclassical three-dimensional drift-diffusion simulations have been performed in [4] and [5] to study the optimized geometry to reduce short channel effects. Still based on semiclassical simulations, devices with a channel length down to $30 \mathrm{~nm}$ have been demonstrated to have near-ideal subthreshold swing and strongly suppressed short channel effects. [8]

Quantum mechanical simulations based on the nonequilibrium Green's function (NEGF) formalism have been performed in [9]-[11], studying fully ballistic transport in SNWT. In those cases, the effective mass approximation has been used. For nanowires with diameter smaller than $4 \mathrm{~nm}$, a full band treatment or atomistic simulations are required, since the effective mass approximation is largely out of its range of validity and is not able to provide accurate estimates of energy gaps and dispersion relations in the longitudinal direction [12]. However, as far as an exploration of the design space is concerned, such approach is still too computationally demanding.

Another issue is that of the appropriate transport model for nanowire transistors. As demonstrated in [13], silicon MOSFETs with effective channel length smaller than $50 \mathrm{~nm}$ will not be fully ballistic, so it is reasonable to assume that even for SNWTs a degree of scattering will be present.

Transport in SNWT is then likely to be in an intermediate regime between fully ballistic and drift-diffusion transport. Dissipative transport can be addressed in a quantum framework as in [9] by means of the Büttiker probes approach, which corresponds to the approximation of a single energy-independent relaxation length, by the definition of self-energies, which account scattering within the NEGF formalism [14], or following an approach similar to that proposed in [15], where drift-diffusion transport in planar MOSFETs is computed in each 2-D subband. We believe the latter approach can provide, with relatively reduced computational resources, relevant information on the degradation of performance with respect to the ballistic case. The actual device will exhibit performance intermediate between the two limiting cases of ballistic and drift-diffusion transport [16].

Here we investigate performance perspectives and scaling issues of triple-gate SNWTs using an in-house developed code based on density functional theory within the effective mass approximation, in which electrostatics and transport are evaluated self-consistently in the 3-D domain, properly considering quantum confinement. 
First, we have considered a device structure with rectangular cross section $(5 \mathrm{~nm} \times 5 \mathrm{~nm})$, in which fully ballistic transport in 1-D subbands is assumed. As a consequence, we have been able to define an upper limit for SNWT performance, deriving significant quantities such as drain-induced barrier lowering (DIBL) and the subthreshold swing $(S)$ as a function of the channel length. Then, the Boltzmann transport equation has been solved in the drift-diffusion approximation in 1-D subbands, in order to study the other limiting case of quasi-equilibrium transport. We shall see that many indexes of device performance are only slightly dependent on the transport model used, and mainly depend on an accurate evaluation of the electrostatics.

\section{BALlistic TRANSPORT: Model AND Results}

The potential profile in the three-dimensional simulation domain obeys the Poisson equation

$$
\nabla[\epsilon(\vec{r}) \nabla \phi(\vec{r})]=-q\left[p(\vec{r})-n(\vec{r})+N_{D}^{+}(\vec{r})-N_{A}^{-}(\vec{r})\right]
$$

where $\phi$ is the electrostatic potential, $\epsilon$ is the dielectric constant, $q$ is the elementary charge, $p$ and $n$ are the hole and electron densities, respectively, $N_{D}^{+}$is the concentration of ionized donors, and $N_{A}^{-}$is the concentration of ionized acceptors. Hole, acceptor, and donor densities are computed in the whole domain with the semiclassical approximation, while the electron concentration in strongly confined regions needs to be computed by solving the Schrödinger equation with density functional theory (DFT), within the effective mass and local density approximations [17], [18]. Since in the considered devices the confinement is strong in the transversal plane $x-y$ and the potential is much smoother in the longitudinal direction $z$, the Schrödinger equation has been adiabatically decoupled in a set of two-dimensional equations with Dirichlet boundary conditions in the $x-y$ plane for each grid-point along $z$, and in a set of one-dimensional equations with open boundary conditions in the longitudinal direction for each 1-D subband. Therefore, transport is assumed to occur in decoupled 1-D subbands.

In particular, the two-dimensional Schrödinger equation for each cross section along $z$ reads

$$
\begin{aligned}
-\left[\frac{\hbar^{2}}{2} \frac{\partial}{\partial x} \frac{1}{m_{\nu x}} \frac{\partial}{\partial x}+\frac{\hbar^{2}}{2} \frac{\partial}{\partial y} \frac{1}{m_{\nu y}} \frac{\partial}{\partial y}\right] \chi_{i \nu}(x, y, z)+ \\
V \chi_{i \nu}(x, y, z)=\varepsilon_{i \nu}(z) \chi_{i \nu}(x, y, z)
\end{aligned}
$$

where $i$ runs over subbands, $\nu$ runs over the three pairs of minima of the conduction band, $\chi_{i \nu}$ and $\varepsilon_{i \nu}$ are the 2-D eigenfunctions and the corresponding eigenenergies computed in the transversal plane at a given $z$, while $m_{\nu x}$ and $m_{\nu y}$ are the effective masses along the $k_{x}$ and $k_{y}$ axis, respectively, on the conduction band pair of minima $\nu$. The confining potential $V$ can be written as $V=E_{C}+V_{\text {exc }}$, where $E_{C}$ is the conduction band and $V_{\text {exc }}$ is the exchange potential within the local density approximation, which reads [19]

$$
V_{\mathrm{exc}}=-\frac{q^{2}}{4 \pi^{2} \varepsilon_{0} \varepsilon_{r}}\left[3 \pi^{3} n(x, y, z)\right]^{\frac{1}{3}} .
$$

First, we consider the optimistic case of fully ballistic transport [20] and thermionic emission in each subband: electrons are in equilibrium with their injecting reservoir (source or drain) and are transmitted with unity probability if their energy in the longitudinal direction is larger than the maximum subband energy $\left(E_{i \nu}^{\max }\right)$, otherwise the transmission coefficient is zero. Clearly, in this context thermionic emission means that source-to-drain tunneling is neglected.

If we define the incomplete Fermi integral of order $-1 / 2$ [21] as

$$
\tilde{\mathcal{F}}_{-\frac{1}{2}}(y, a, b)=\int_{a}^{b} \frac{1}{\sqrt{E}} \frac{1}{1+\exp (E-y)} d E
$$

the electron concentration can be expressed as

$$
\begin{aligned}
n= & \sum_{i} \sum_{\nu=1}^{3} N_{1 \mathrm{D}_{\nu}}\left|\chi_{i \nu}(x, y, z)\right|^{2} \\
& \times \tilde{\mathcal{F}}_{-\frac{1}{2}}\left(\frac{E_{F_{l}}-\varepsilon_{i \nu}(z)}{K_{B} T}, 0, \frac{E_{i \nu}^{\max }-\varepsilon_{i \nu}(z)}{K_{B} T}\right) \\
& +\frac{N_{1 \mathrm{D}_{\nu}}}{2}\left|\chi_{i \nu}(x, y, z)\right|^{2} \\
& \times\left[\tilde{\mathcal{F}}_{-\frac{1}{2}}\left(\frac{E_{F_{S}}-\varepsilon_{i \nu}(z)}{K_{B} T}, \frac{E_{i \nu}^{\max }-\varepsilon_{i \nu}(z)}{K_{B} T},+\infty\right)\right. \\
& \left.\quad+\tilde{\mathcal{F}}_{-\frac{1}{2}}\left(\frac{E_{F_{D}}-\varepsilon_{i \nu}(z)}{K_{B} T}, \frac{E_{i \nu}^{\max }-\varepsilon_{i \nu}(z)}{K_{B} T},+\infty\right)\right]
\end{aligned}
$$

where $K_{B}$ is Boltzmann's constant, $N_{1 \mathrm{D}_{\nu}}$ is the equivalent onedimensional density of states in the $\nu$-th pair of minima of the conduction band, with effective mass $m_{\nu z}$ in the longitudinal direction $z$

$$
N_{1 \mathrm{D}_{\nu}}=\frac{2}{\pi}\left(\frac{2 m_{\nu z} K_{B} T}{\hbar^{2}}\right)^{\frac{1}{2}}
$$

and $E_{F_{l}}=E_{F_{S}}\left(E_{F_{D}}\right)$ if the coordinate $z$ is between the subband maximum and the source (drain).

The source-to-drain current $I_{\mathrm{DS}_{i \nu}}$ in the generic $i$-th subband and in the $\nu$-th pair of minima $\varepsilon_{i \nu}$ reads

$$
I_{D S_{i \nu}}=2 \frac{q}{\pi \hbar} \int_{\varepsilon_{i \nu}}^{\infty} \tau_{i \nu}(E)\left[f_{S}(E)-f_{D}(E)\right] d E
$$

where $\hbar$ is reduced Planck's constant, and $f_{S}$ and $f_{D}$ are the Fermi-Dirac functions with the Fermi level of the source and the drain, respectively, while $\tau_{i \nu}(E)$ is the transmission coefficient.

In order to consider the impact of quantum tunneling on device performance, once computed the self-consistent potential in the thermionic case, we have also computed the transmission coefficient in each 1-D subband by means of the transmission matrix formalism. Here we have made the assumptions that tunneling electrons have a negligible effect on the potential, and do not include them in the self-consistent scheme. Such an approximation can limit the accuracy especially for shorter devices, quantitatively affecting the difference between the results of the quantum and the semiclassical model. From here on, this case will be referred as the quantum fully ballistic case.

From a numerical point of view, the Poisson and Schrödinger equations have been solved with a predictor/corrector scheme similar to that proposed in [22].

In Fig. 1 the flow-chart of the implemented algorithm is shown. Starting from an initial solution, the Schrödinger 


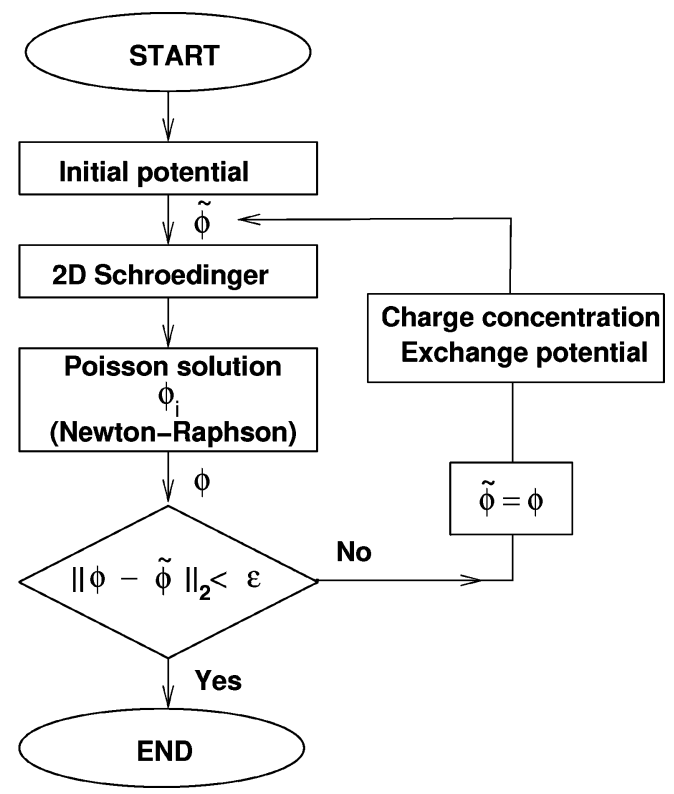

Fig. 1. Flow-chart of the self-consistent 3-D Poisson-Schrödinger solver.

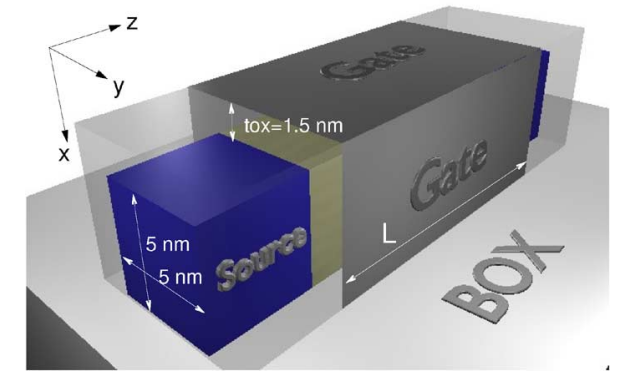

Fig. 2. Three-dimensional structure of the simulated triple-gate SNWT.

equation is solved for the potential $\tilde{\phi}$. The nonlinear Poisson equation is then computed by means of the Newton-Raphson (NR) algorithm. In particular, at each NR step, the computed eigenfunctions are kept constant, while the term $\varepsilon_{i \nu}(z)$ in (5) is changed to $\varepsilon_{i \nu}(z)+q\left[\phi_{i}(x, y, z)-\tilde{\phi}(x, y, z)\right]$, where $\phi_{i}$ is the potential at the $i$-th NR step. When the NR algorithm converges, the solution $\phi$ is compared with $\tilde{\phi}$ : if the two norm of the difference is smaller than a predetermined value the algorithm stops, otherwise another cycle is performed with $\tilde{\phi}=\phi$.

In Fig. 2 the simulated triple-gate SNWT structure is shown. The oxide thickness is $1.5 \mathrm{~nm}$, and the channel length $L$ ranges from 7 to $25 \mathrm{~nm}$. The wire is $p$-doped with $N_{A}=10^{17} \mathrm{~cm}^{-3}$ and the source and drain are doped with $N_{D}=10^{20} \mathrm{~cm}^{-3}$. Degenerate statistics is considered in the wire. The gate is metallic and its workfunction is chosen to adjust the threshold voltage of all structures to $0.2 \mathrm{~V}$ (the threshold voltage is defined here as the gate voltage required to have $I_{D S}=0.1 \mu \mathrm{A}$ for $V_{D S}=$ $0.5 \mathrm{~V})$.

In Fig. 3 an isosurface of the electron concentration for a device with channel $15 \mathrm{~nm}, V_{G S}=V_{D S}=0.5 \mathrm{~V}$ is shown: one can clearly see that there is a volume inversion, and that the device is in the saturation regime, as confirmed by the constriction in correspondence of the drain.

In Fig. 4 the transfer characteristics are plotted for $L=25 \mathrm{~nm}$ and $L=7 \mathrm{~nm}$ for a drain-to-source voltage $V_{\mathrm{DS}}=0.5 \mathrm{~V}$. For

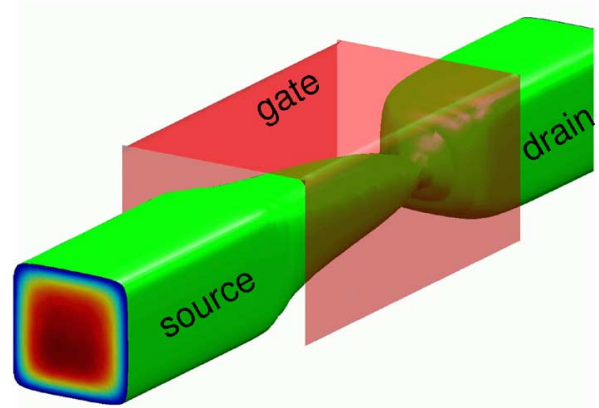

Fig. 3. Electron density isosurface $\left(n=1.4 \times 10^{19} \mathrm{~cm}^{-3}\right)$ computed for the SNWT with channel length equal to $15 \mathrm{~nm}$, for a gate voltage $V_{G S}=0.5 \mathrm{~V}$ and a source-to-drain voltage $V_{D S}=0.5 \mathrm{~V}$.

$\square \cdots \square$ quantum fully ballistic $\diamond \diamond$ thermionic emission

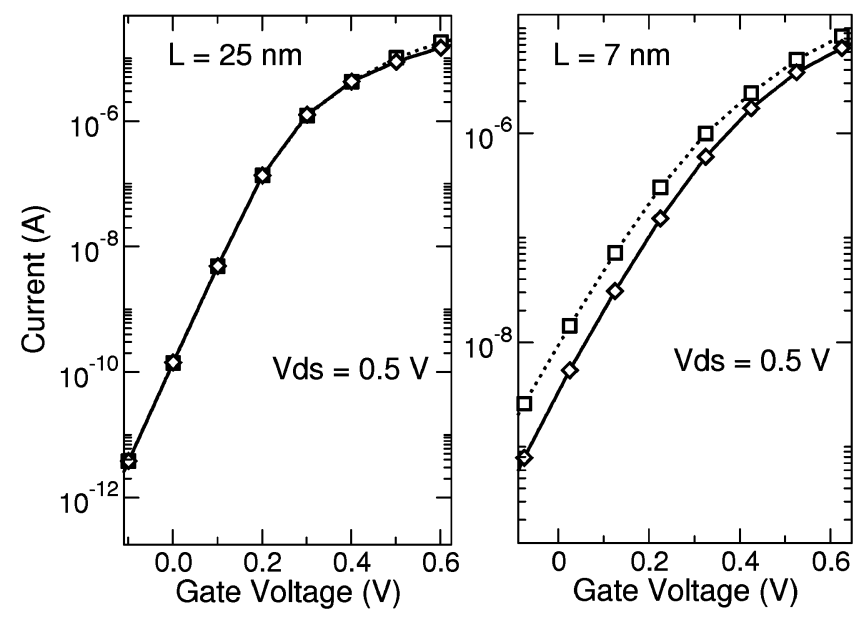

Fig. 4. Transfer characteristics computed for $V_{\mathrm{DS}}=0.5 \mathrm{~V}$, for the $L=25 \mathrm{~nm}$ and $L=7 \mathrm{~nm}$ devices.

the longer device, in subthreshold, the quantum fully ballistic and the thermionic emission models give almost the same results, while differences become relevant for the shorter device. For a channel length of $7 \mathrm{~nm}$ source-to-drain tunneling is significant both in subthreshold and in strong inversion conditions, while for $L=25 \mathrm{~nm}$ tunneling is quantitatively relevant only in strong inversion. Such behavior is clearer if we consider $I_{\mathrm{on}}$ and $I_{\text {off }}$ as a function of $L$, shown in Fig. 5(a) and (b), respectively, where $I_{\mathrm{off}}$ is defined as the current obtained for $V_{\mathrm{DS}}=0.5 \mathrm{~V}$ and a gate voltage $V_{\mathrm{GS}}=0 \mathrm{~V}$, and $I_{\mathrm{on}}$ as the current obtained for $V_{\mathrm{DS}}=0.5 \mathrm{~V}$ and $V_{\mathrm{GS}}=0.5 \mathrm{~V}$.

Quantum tunneling gives a contribution dependent on $L$, and significant for the $I_{\mathrm{on}}$ already for $L=25 \mathrm{~nm}$.

The reason can be understood considering that, for example, for $L=25 \mathrm{~nm}$ the product of the transmission probability times the occupation factor $\left[\tau_{11}(E) f_{S}(E)\right]$ has the energy dependence shown in Fig. 6, for thermionic emission (thin line) and quantum tunneling (thick line).

The subband current is proportional to the area below the curve. Quantum tunneling adds a contribution that is proportional to the difference between the area $a$ and the area $b$. The shape of the subband peak, depends essentially on the vertical electrostatics, and determines $a$ and $b$. In addition, as shown in Fig. 7, the shape only slightly depends on $L$, since even for the 


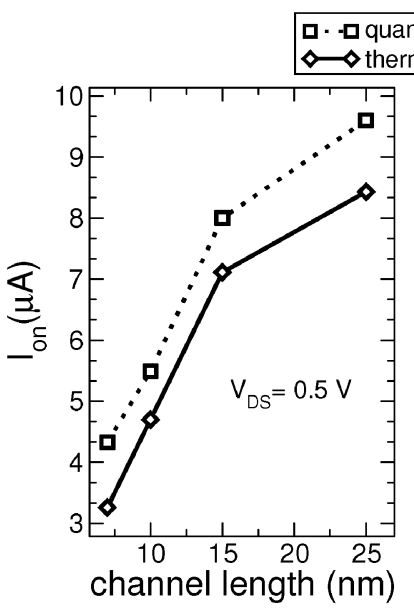

a)

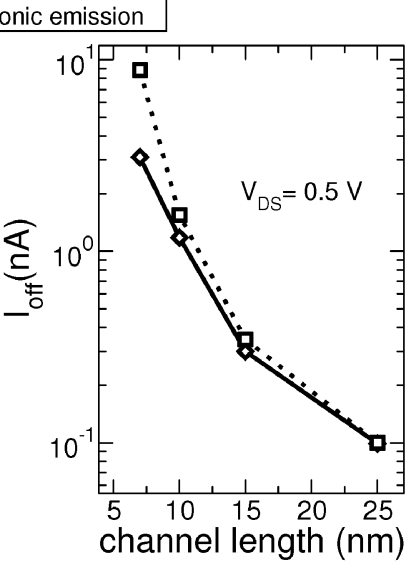

b)
Fig. 5. (a) $I_{\text {on }}$ and (b) $I_{\text {off }}$ currents as a function of the channel length, for the quantum fully ballistic and thermionic emission transport models.

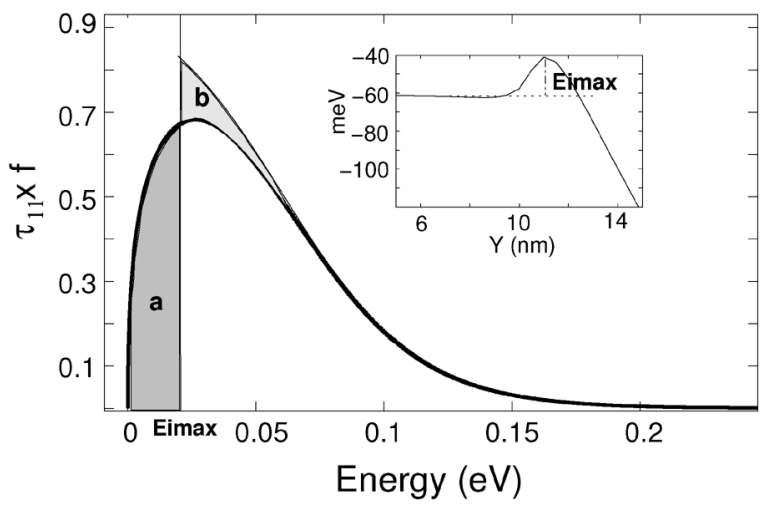

Fig. 6. Argument of integral in $(7): \tau_{11}(E) f_{S}(E)$. The upper curve is the argument in the thermionic case, while the lower curve the argument in the quantum case. Tunneling current is larger than the thermionic current, since the area $a$ is larger than the area $b$. The inset refers to $V_{D S}=0.5 \mathrm{~V}$ and $V_{G S}=0.8 \mathrm{~V}$.

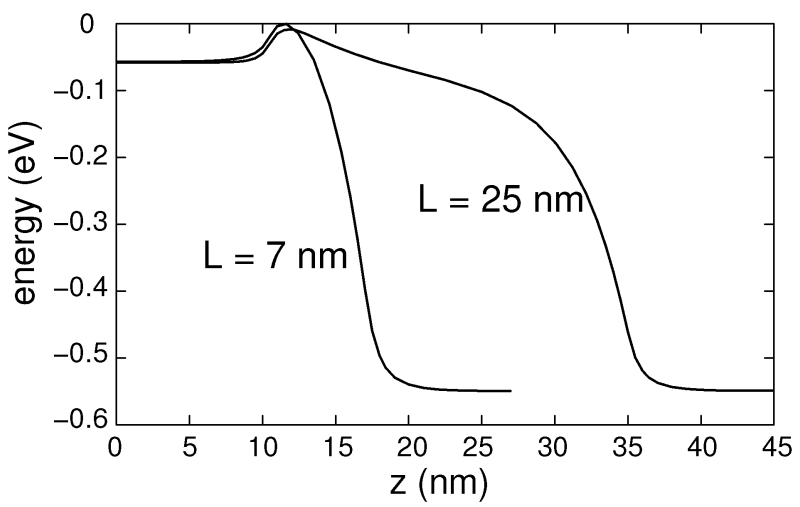

Fig. 7. Comparison between the first subband computed for the $L=7 \mathrm{~nm}$ and $L=25 \mathrm{~nm}$ devices for $V_{G S}=0.5 \mathrm{~V}$ and $V_{D S}=0.5 \mathrm{~V}$ : the shapes of the subband peak are almost the same for the two considered cases, which in turns give the same tunneling currents.

shorter and longer considered devices, the top of the barrier has almost the same profile.

On the other hand, $I_{\text {off }}$ is affected by quantum tunneling only for $L=10 \mathrm{~nm}$ and below, and is extremely sensitive to the channel length. Such dependence is mainly due to the degradation of the subthreshold slope $\mathrm{S}$ with decreasing length [shown in Fig. 8(a)].

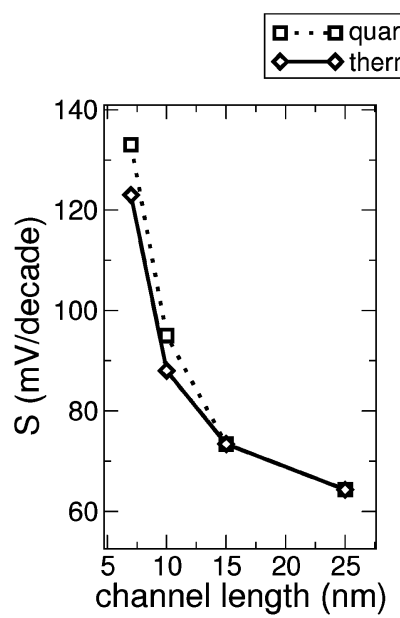

a)

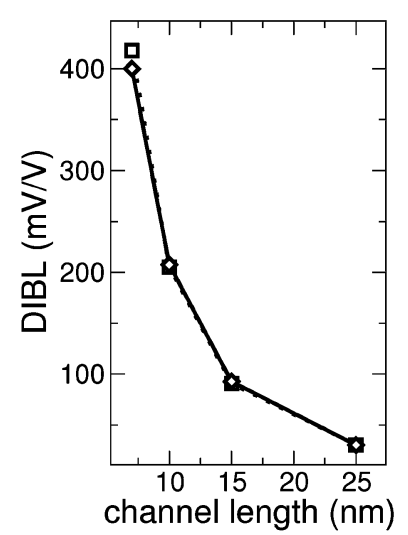

b)
Fig. 8. (a) Subthreshold swing and (b) drain-induced barrier lowering as a function of the channel length, for the quantum fully ballistic and thermionic emission transport models.

In addition, once fixed the threshold voltage, the larger gatechannel capacitative coupling for longer devices makes the $I_{\text {on }}$ increase as the channel length is increased, while short channel effects degrade $I_{\text {off }}$ in shorter devices. Indeed, as can be seen in Fig. 8(b), down to $15 \mathrm{~nm}, S$ is very good $(<70 \mathrm{mV} / \mathrm{dec})$, as well as DIBL [Fig. 8(b)], while for smaller $L, S$ is degraded by charge sharing, with a consequent degradation of the $I_{\text {off }}$.

\section{DRIFT-DIFFUSION TRANSPORT IN 1-D SUBBANDS: MODEL AND RESULTS}

As discussed before, dissipative transport can be addressed with simple models based on the solution of the continuity equation in the drift-diffusion approximation in each 1-D subband. In this way it is possible to define a lower limit for device performance.

In particular, the current density for electrons in the $i$-th subband and the $\nu$-th conduction band pair of minima reads

$$
J_{n_{i \nu}}=q \mu_{n_{i \nu}} n_{1 \mathrm{D}_{i \nu}} \frac{\partial \varepsilon_{i \nu}}{\partial z}+q D_{n_{i \nu}} \frac{\partial n_{1 \mathrm{D}_{i \nu}}}{\partial z}
$$

where $n_{1 \mathrm{D}_{i \nu}}$ is the electron density per unit length, and $\mu_{n_{i \nu}}$ and $D_{n_{i \nu}}$ are the mobility and the diffusion coefficient for electrons, respectively.

For what concerns mobility, here we have assumed a very simple model, which simply includes velocity saturation by means of the Caughey-Thomas model [23] in the case of Maxwell-Boltzmann statistics

$$
\mu_{n}=\frac{\mu_{0}}{\left[1+\left(\frac{\mu_{0} \mathcal{E}_{1}}{v_{\mathrm{sat}}}\right)^{\gamma}\right]^{\frac{1}{\gamma}}}
$$

where $\mathcal{E}_{l}$ is the local longitudinal field, $v_{\text {sat }}$ is the velocity saturation $\left(v_{\mathrm{sat}}=1.1 \times 10^{7} \mathrm{~cm} / \mathrm{s}\right), \gamma$ is a constant fitting parameter ( $\gamma=2$ is a common value for electrons at room temperature), while $\mu_{0}$ has been taken constant.

We are aware that a more accurate approach would require a deeper insight into silicon nanowire mobility, including surface, and electron-phonon scattering, but we believe that the 


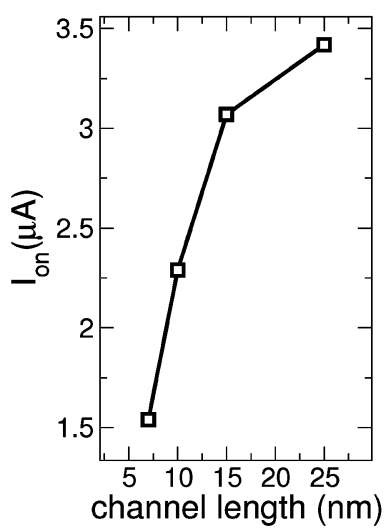

a)

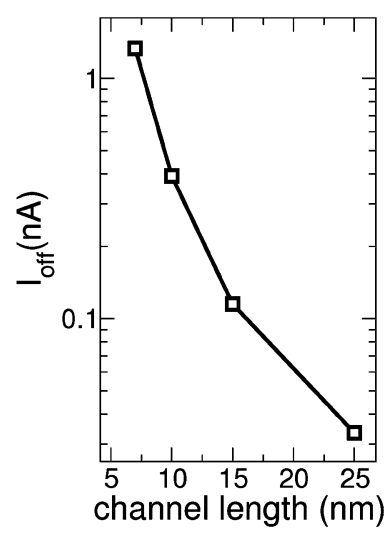

b)
Fig. 9. (a) $I_{\text {on }}$ and (b) $I_{\text {off }}$ currents computed by means of the drift-diffusion model, as a function of the channel length.

model assumed can still give relevant information on device behavior. Here, rather than focus on accurate mobility models for SNWTs, we want to define a simulation approach that accounts for drift-diffusion transport in 1-D subbands, representing a lower limit for device performance. Consistently we use (9) for the mobility of each subband, and we compute the corresponding diffusion coefficient using Einstein's relation, instead of the relationship that would be suitable for Fermi-Dirac statistics. Given the modular structure of the code, extension to more complex mobility models is straightforward.

Once computed $n_{1 \mathrm{D}_{i \nu}}$, the three-dimensional electron density $(n)$ reads

$$
n=\sum_{i} \sum_{\nu=1}^{3}\left|\chi_{i \nu}\right|^{2} n_{1 \mathrm{D}_{i \nu}} .
$$

From a numerical point of view, a continuity equation for each 1-D subbands is solved using the Scharfetter and Gummel scheme [24]. Inter-subband scattering is neglected. In possible extension, it could be included as a generation-recombination term. To improve convergence of the Newton-Raphson scheme, we have modified (10), inserting a Maxwell-Boltzmann-like correcting factor

$$
n=\sum_{i} \sum_{\nu=1}^{3}\left|\chi_{i \nu}\right|^{2} n_{1 \mathrm{D}_{i \nu}} \exp \left[\frac{q(\phi-\tilde{\phi})}{K_{B} T}\right] .
$$

Obviously at convergence, (11) reduces to (10).

In Fig. 9 the $I_{\text {on }}$ and $I_{\text {off }}$ are plotted as a function of the channel length, computed by means of the drift-diffusion model. Both $I_{\mathrm{on}}$ and $I_{\mathrm{off}}$ show the same behavior observed for the ballistic models as the channel length varies, while are smaller by almost a factor two as compared to ballistic currents.

In Fig. 10 instead the subthreshold swing and the DIBL as a function of $L$ are shown. Even in the drift-diffusion case, $S$ is very good down to $15 \mathrm{~nm}$.

Conversely, drain-induced barrier lowering is much higher for ballistic than for drift-diffusion transport. In the former case, current is essentially controlled by the subband peak, while in the latter, it is roughly dependent on the whole region between the source and the subband peak that is farther from the drain.

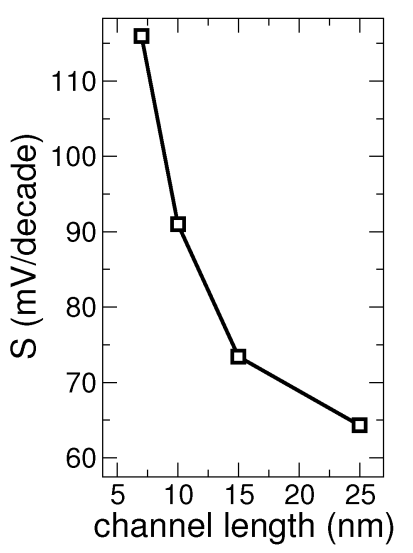

a)

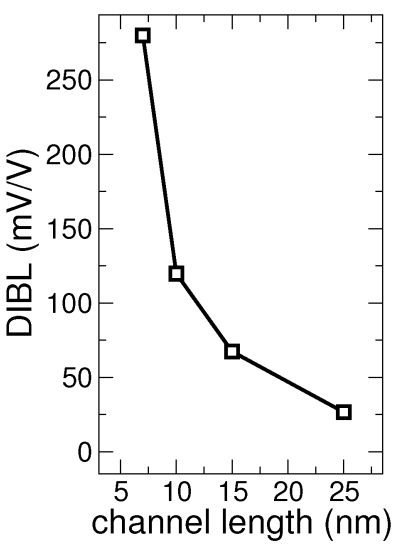

b)
Fig. 10. (a) Subthreshold swing and (b) drain-induced barrier lowering computed by means of the drift-diffusion model, as a function of the channel length.

\section{CONCLUSIONS}

We have investigated the electrical properties of SNWTs with detailed quantum simulations considering ballistic transport, both in the quantum and in the thermionic emission case, in order to evaluate the upper limiting cases for the performance of triple-gate SNWTs. SNWTs with diameter $5 \times 5 \mathrm{~nm}$ offer promising scaling perspectives down to $10 \mathrm{~nm}$ channel lengths, predicted at the end of the ITRS Roadmap.

A particularly interesting and unexpected result is that the impact of quantum tunneling is significant in strong inversion also in longer devices, where source-to-drain tunneling in subthreshold is negligible, because it depends essentially on the vertical electrostatics rather than on $L$.

As expected, in a drift-diffusion transport regime, $I_{\text {on }}$ is degraded by a factor close to 2 with respect to the ballistic case, while the $I_{\mathrm{on}} / I_{\mathrm{off}}$ ratio is almost independent of the transport model. On the other hand, short channel effects exhibit a small dependence on the transport regime.

Finally, we want to stress the fact that our nanowire transistor simulation tool represents a significant advantage with respect to the state of the art: it allows to address generic three-dimensional structures with no spatial symmetry, to properly take into account the impact of quantum confinement on the density of states through density functional theory, and to treat both ballistic and dissipative transport in 1-D subbands. We believe that the chosen approximations ensure a very good tradeoff between accuracy and computational resources required.

Further extensions, such as introducing limited intersubband scattering via a generation-recombination term, or including a more sophisticated mobility model, or even an energy-balance model per subband, can be done substantially without modifying the code structure.

\section{REFERENCES}

[1] International Technology Roadmap for Semiconductor 2003, Semiconductor Industry Association [Online]. Available: http://www. public.itrs.net

[2] Y. Cui, Z. Zhong, D. Wang, W. U. Wang, and C. M. Lieber, "High performance silicon nanowire field effect transistors," Nano Lett., vol. 3, pp. 149-152, 2003.

[3] X. Duan, Y. Huang, Y. Cui, J. Wang, and C. M. Lieber, "Indium phosphide nanowires as building blocks for nanoscale electronic and optoelectronics devices," Nature, vol. 409, pp. 66-69, 2001. 
[4] J. T. Park and J.P. Colinge, "Multiple-gate SOI MOSFETs: Device design guidelines," IEEE Trans. Electron Devices, vol. 49, no. 12, pp. 2222-2229, Dec. 2002.

[5] J. P. Colinge, J. T. Park, and C. A. Colinge, "SOI devices for sub-0.1 $\mu \mathrm{m}$ gate lengths," in Proc. Int. Conf. Microelectronics (MIEL 2002), pp. 109-113.

[6] T. Saito, T. Saraya, T. Inukai, H. Majimi, T. Nangumo, and Hiramoto, "Suppression of short channel effect in triangular parallel wire channel MOSFETs," IEICE Trans. Electron, vol. E85-C, no. 5, pp. 1073-1080, May 2002

[7] B. Y. , L. Chang, S. Ahmed, H. Wang, S. Bell, C. Y. Yang, C. Tabery, C. Ho, Q. Xiang, T. J. King, J. Bokor, C. Hu, M. R. Lin, and D. Kyser, "FinFET scaling to $10 \mathrm{~nm}$ gate length," in IEDM Tech. Dig., 2002, pp. 251-254.

[8] B. S. Doyle, S. Datta, M. Doczy, S. Hareland, B. J. Kavalieros, T. Linton, A. Murthy, R. Rios, and R. Chau, "High performance fully-depleted tri-gate CMOS transistors," IEEE Trans. Electron Devices, vol. 24, no. 4, pp. 263-265, Apr. 2003.

[9] J. Wang, E. Polizzi, and M. Lundstrom, "A three-dimensional quantum simulation of silicon nanowire transistors with the effective-mass approximation," J. Appl. Phys., vol. 96, pp. 2192-2203, 2004.

[10] M. Bescond, K. Nehari, J. L. Autran, N. Cavasillas, D. Munteanu, and M. Lannoo, "3D quantum modeling and simulation of multiple-gate nanowire MOSFETs," in IEDM Tech. Dig., 2004, pp. 617-620.

[11] J. Wang, E. Polizzi, and M. Lundstrom, "A computational study of ballistic silicon nanowire transistor," in IEDM Tech. Dig., 2003, pp. 29.5.1-29.5.4.

[12] Y. Zheng, C. Rivas, R. Lake, K. Alam, T. B. Boykin, and G. Klimeck, "Electronic properties of silicon nanowires," IEEE Trans. Electron Devices, vol. 52, no. 6, pp. 1097-1103, Jun. 2005.

[13] A. Lochtefeld and D. A. Antoniadis, "On experimental determination of carrier velocity in deeply scaled NMOS: How close to the thermal limit?," IEEE Electron Device Lett., vol. 22, no. 2, pp. 95-97, Feb. 2001.

[14] S. Jin, Y. J. Park, and H. S. Min, "A three-dimensional simulation of quantum transport in silicon nanowire transistor in the presence of electron-phonon interactions," J. Appl. Phys., vol. 99, pp. 123719-10-, 2006.

[15] G. Curatola, G. Doornbos, J. Loo, Y. V. Ponomarev, and G. Iannaccone, "Detailed modeling of sub-100-nm MOSFETs based on Schrödinger DD per subband and experiments and evaluation of the performance gap to ballistic transport," IEEE Trans. Electron Devices, vol. 52, no. 8, pp. 1851-1858, Aug. 2005.

[16] S. Jin, Y. J. Park, and H. S. Min, "Influence of electron-phonon interactions on the electronic transport in nanowire transistors," in Proc. Int. Conf. Simulation of Semiconductor Processes and Devices, 2006, pp. 35-38.

[17] P. Hohenberg and W. Kohn, "Ihnomogeneous electron gas," Phys. Rev., vol. 136, pp. B864-B871, 1964.

[18] W. Kohn and L. J. Sham, "Self consistent equations including exchange and correlation effects," Phys. Rev., vol. 140, pp. A1133-A1138, 1965.

[19] J. C. Slater, "A simplification of the Hartree-Fock method," Phys. Rev., vol. 81, pp. 385-390, 1951.

[20] K. Natori, "Ballistic metal-oxide-semiconductor field effect transistor," J. Appl. Phys., vol. 76, pp. 4879-4890, 1994.
[21] M. Goano, "Algorithm 745: Computation of the complete and incomplete Fermi-Dirac integral," ACM Trans. Math. Softw., vol. 21, pp. 221-232, Sep. 1995.

[22] A. Trellakis, A. T. Galick, A. Pacelli, and U. Ravaioli, "Iteration scheme for the solution of the two-dimensional Schrdinger-Poisson equations in quantum structures," J. Appl. Phys, vol. 81, pp. 7880-7884, Mar. 1997.

[23] D. M. Caughey and R. E. Thomas, "Carrier mobilities in silicon empirically related to doping and field," IEEE Proc., vol. 55, pp. 2192-2193, 1967.

[24] D. L. Scharfetter and D. L. Gummel, "Large signal analysis of a silicon read diode oscillator," IEEE Trans. Electron Devices, vol. ED-16, no. 1, pp. 64-77, Jan. 1969.

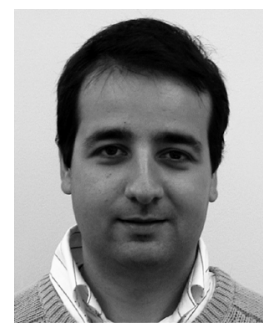

Gianluca Fiori received the degree in electronic engineering and the $\mathrm{Ph} . \mathrm{D}$. degree from the University of Pisa, Italy, in 2001 and 2005, respectively.

In autumn 2002, he was in SILVACO International developing quantum models, which are currently implemented in the commercial simulator ATLAS by SILVACO. In summer 2004 and 2005, he was at Purdue University, working on models for the simulation of transport in nanoscale devices. $\mathrm{He}$ is currently working at Dipartimento di Ingegneria dell'Informazione, at Pisa University, holding a postdoc position. His main field of activity is the development of models and codes for the simulations of ultrascaled semiconductor devices. Further information is available at http://www.monteverdi.iet.unipi.it/ fiori.

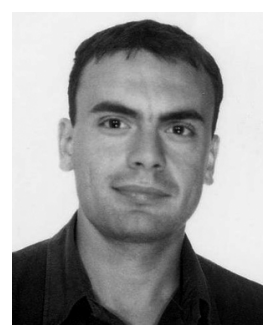

Giuseppe Iannaccone received the M.S. and Ph.D. degrees in electrical engineering from the University of Pisa, Italy, in 1992 and 1996, respectively.

In 1996 he took a permanent position as researcher with the Italian National Research Council and the same year he obtained a faculty position in the Electrical Engineering Deparment of the University of Pisa, first as an Assistant Professor, then, since January 2001, as Associate Professor of Electronics. $\mathrm{He}$ has authored and coauthored more than 110 papers published in peer-reviewed journals and more than 70 papers in proceedings of international conferences. His interests include transport and noise in nanoelectronic and mesoscopic devices, development of device modeling and TCAD tools, and the design of extremely low power circuits and systems for RFID and ambient intelligence scenarios.

Prof. Iannaccone has coordinated a few European and national projects involving multiple partners, and has acted as principal investigator in several research projects funded by public agencies at the European and national level, and by private organizations. He is in the technical committee of few international conferences and serves as a referee for the leading journals in the fields of condensed matter physics, device electronics, and circuit design. 